EXTENDED REPORT

\title{
A prospective study of pregnant patients with rheumatoid arthritis and ankylosing spondylitis using validated clinical instruments
}

\author{
M Østensen, L Fuhrer, R Mathieu, M Seitz, P M Villiger
}

Ann Rheum Dis 2004;63:1212-1217. doi: 10.1136/ard.2003.016881

See end of article for authors' affiliations

\section{Correspondence to:}

Professor M Østensen Department of

Rheumatology and Clinical Immunology and Allergy, University Hospital, $\mathrm{CH}$ 3010 Bern, Switzerland; monika.oestensen@ insel.ch

Accepted 1 December 2003

\begin{abstract}
Objective : To analyse the disease course of patients with rheumatoid arthritis (RA) and ankylosing spondylitis (AS) during and after pregnancy by validated clinical instruments for measurement of disease activity, and assess their usefulness in pregnant patients.

Methods: Included were 10 patients with RA and 9 with AS (10 pregnancies). Clinical examination and blood/urine sampling was performed before conception, at each trimester, and weeks 6, 12, and 24 post partum. Assessment of RA was by the RA Disease Activity Index (RADAl), the 44 joint count, and the Health Assessment Questionnaire; assessment of AS by the Bath Ankylosing Spondylitis Activity Index (BASDAI), the Dougados Functional and Articular Index, and a night pain index. Common for all patients were the patient's and physician's global assessment.

Results : Most patients with RA showed sustained or increased improvement of disease activity during pregnancy. Higher disease activity scores were found in the patients with AS with a frequent increase of disease activity in the second trimester and mitigation of symptoms in the third trimester. Analysis specifically for the patient's assessment of pain showed continuously higher pain scores in the patients with AS than in those with RA. Rank correlation showed good to moderate correlation between most clinical measurements and RADAI or BASDAI, respectively. Functional indices were confounded by physiological changes of late pregnancy.

Conclusion: RA can be monitored during and after pregnancy by the swollen joint count and RADAI without interference from pregnancy related symptoms, whereas usual measures of disease activity are not always applicable in pregnant patients with AS.
\end{abstract}

regnancy has been recognised as a disease modifying factor in several rheumatic diseases. The effects of pregnancy on rheumatoid arthritis (RA) have been reported as mostly beneficial, ${ }^{1}$ whereas disease symptoms of ankylosing spondylitis (AS) have been recorded as largely unaltered. ${ }^{2}$ Retrospective studies comprising a total of 345 pregnancies indicated that arthritis improved in about $75 \%$ (range $54-83 \%$ ) of patients with RA. ${ }^{3}$ Six detailed prospective studies comprising 177 pregnancies found improvement or remission in about two thirds. ${ }^{4-7}$ According to retrospective and prospective studies, disease recurs in most patients within 3-4 months of delivery. In contrast with RA, prospective and retrospective studies have found that the activity of AS is unchanged by pregnancy. ${ }^{4}{ }^{8}$ Improvement of disease symptoms has been reported to occur preferentially in patients with a history of peripheral arthritis or accompanying disease like inflammatory bowel disease or psoriasis.' Aggravation of AS within 6 months after delivery has been reported for $60-90 \%$ of patients. ${ }^{9}$

Relief of arthritis is different from improvement documented by validated instruments for disease activity. In the retrospective studies of pregnant patients with RA and AS, assessment of disease activity has relied on the patient's recall, which at best gives an overall estimate of disease activity. It cannot depict the precise timing and extent of change of disease activity and thereby excludes an analysis of the factors which possibly are involved in the induction of change. Prospective studies have assessed the disease activity of RA by various methods, including the Camp index, ${ }^{10}$ the Health Assessment Questionnaire (HAQ), ${ }^{11}$ the Arthritis Impact Measurement Scale, ${ }^{12}$ self reported pain, swelling, and morning stiffness, ${ }^{13}$ the Ritchie index, the number of swollen joints, ${ }^{6}$ and drug treatment. Pre-pregnancy assessment was included in two studies, the postpartum follow up varied between 6 weeks and 1 year. ${ }^{4-7} 1415$

Previous studies of pregnancies in patients with AS have reported unchanged disease activity during pregnancy. ${ }^{2}$ However, this observation was based on the patient's overall assessment in retrospective studies. In prospective studies, spinal tenderness, spinal mobility, functional class, duration of morning stiffness, and nocturnal pain were recorded. ${ }^{48}$ None of the previous studies on pregnancy in RA or AS has been based on clinical instruments used at present to assess disease activity in clinical trials nor have any of the instruments applied been validated for use during pregnancy.

In the present 64 week prospective study, we chose to regard pregnancy as a disease modifying agent and to measure disease activity in patients with RA and AS by instruments which have been validated in clinical trials. We did this by analysing in detail the clinical response pattern of both groups of patients and assessed the usefulness of several clinical instruments for monitoring pregnant patients.

\section{PATIENTS AND METHODS}

The study was approved by the ethical committee of the University of Berne. The patients were recruited at the

Abbreviations: AS, ankylosing spondylitis; ASAS, Ankylosing Spondylitis Assessment; BASDAl, Bath Ankylosing Spondylitis Activity Index; CRP, C reactive protein; DAl, Dougados Articular Index; DFI, Dougados Functional Index; DMARDs, disease modifying antirheumatic drugs; ESR, erythrocyte sedimentation rate; $\mathrm{HAQ}$, Health Assessment Questionnaire; NSAIDs, non-steroidal anti-inflammatory drugs; RA, rheumatoid arthritis; RADAl, RA Disease Activity Index 
Table 1 Clinical characteristics of the 10 patients with rheumatoid arthritis (RA) and nine patients with ankylosing spondylitis (AS) at entry into the study

\begin{tabular}{lll}
\hline Characteristics & RA & AS \\
\hline Age (years) & $28(25-37)$ & $31(26-38)$ \\
Disease duration in years (range) & $3(0-10)$ & $9(2-13)$ \\
Smoking & 2 & 1 \\
Previous DMARD treatment & 10 & 1 \\
Previous prednisone treatment & 5 & 2 \\
Rheumatoid factor positive & 6 & ND* \\
ANA positive & 5 & ND \\
HLA-B27 positive & ND & 7 \\
\hline
\end{tabular}

*DMARD, disease modifying antirheumatic drug; ANA, antinuclear antibody; ND, not done.

Department of Rheumatology, University Hospital, Berne, after informed consent. Included in the study were 10 patients fulfilling the American College of Rheumatology criteria for $\mathrm{RA}^{16}$ and nine patients (with 10 pregnancies) fulfilling the European Spondylarthropathy Study Group for AS. ${ }^{17}$ Patients with AS all had radiologically confirmed arthritis of the sacroiliac joints, and three of them had a history of peripheral arthritis in hips, knees, or ankles. Table 1 gives the disease characteristics and drug treatment of all patients at study entry.

Drugs allowed during pregnancy were antimalarial agents, sulfasalazine, or low dose prednisone ( $<10 \mathrm{mg}$ prednisone/ day) and non-steroidal anti-inflammatory drugs (NSAIDs) until week 32 of gestation. The same drugs were reintroduced after delivery at the first sign of reactivation of disease.

Clinical examination and blood/urine sampling of pregnant patients was performed according to a standard protocol at the following times: once within 6 months before conception ( 12 patients), once between gestational weeks 9 and 11 , once between gestational weeks 20 and 22, once between gestational weeks 30 and 34, and 6, 12, and 24 weeks after delivery.

Clinical disease activity was monitored by physical examination and by validated instruments for RA and AS. Patients with RA were assessed by the RA Disease Activity Index (RADAI), ${ }^{18}$ the 44 joint count, ${ }^{19}$ and the HAQ. ${ }^{11}$ The RADAI score ranges from 0 (no disease activity) to 10 (maximum disease activity). A relevant change is indicated by a difference of 1.0-1.5. The HAQ ranges from 0 (no functional impairment) to 3 (maximum functional impairment). The minimal relevant change is 0.17.

Patients with AS were assessed by the Bath Ankylosing Spondylitis Activity Index (BASDAI), ${ }^{20}$ the Dougados Functional (DFI) and Articular Index (DAI) ${ }^{21}$, and a night pain index. ${ }^{22}$ The BASDAI ranges from 0 to 10 , the DFI from 0 to 60 , the DAI from 0 to 40 , and the night pain index from 0 to 3. Assessments common for all patients were the patient's and the physician's global assessment $(100 \mathrm{~mm}$ visual analogue scale).

Disease activity was chosen as the outcome measure for pregnancy and post partum in RA and AS. Remission in RA was defined according to modified remission criteria (fatigue and erythrocyte sedimentation rate (ESR) omitted because of pregnancy $)^{23}$ : morning stiffness $<15$ minutes, no soft tissue swelling in joints or tendon sheaths, no joint tenderness or pain on motion, no (need for) drug treatment. Active disease in RA was defined as present when modified remission criteria did not apply. Ankylosing Spondylitis Assessment (ASAS) response criteria were used to measure a $20 \%$ improvement in patients with AS. $^{24}$ Active disease in AS was defined as a BASDAI of $>2$.
Routine laboratory investigations were $\mathrm{C}$ reactive protein (CRP), haemoglobin, leucocyte and thrombocyte count, and urine analysis.

\section{Statistical analysis}

As the primary end point, changes of disease activity scores during the observation period were assessed for each cohort by the Friedmann test with Bonferroni correction. The course of clinical assessments was depicted by calculating the median and the 95\% confidence interval for each score according to the method of the Hodges-Lehmann estimate. Correlations between disease activity scores were calculated by the rank correlation coefficient. Correlations of $0.30-0.70$ were considered as moderate to substantial correlations, and coefficients $<0.30$ were considered as weak correlations.

\section{RESULTS}

Table 1 gives clinical characteristics of the patients at study entry. One patient with RA had a pre-pregnancy visit, but miscarried spontaneously at week 8 and did not meet for further controls. The other nine patients with RA and nine with AS (with 10 pregnancies) met for the pregnancy time points and 6, 12, and 24 weeks post partum. The numbers of patients with RA and with AS included at each time (RA/AS) were: $6 / 6$ at the pre-pregnancy visit within 6 months of the index pregnancy, 9/9 at the first, $9 / 10$ at the second and the third trimester, $9 / 10$ at 6 weeks, and 9/9 at 12 and 24 weeks post partum.

\section{Disease activity during and after pregnancy Patients with RA}

Figure 1 shows the disease course of all patients with RA by the swollen joint count (A), the RADAI (B), and the patient global score (C). One patient with RA with persistent low back pain during the observation period showed high patient assessment scores (figs $\mathrm{IB}$ and $\mathrm{C}$ ) despite remission documented by the joint count (fig 1A). According to modified remission criteria, three patients with RA were in remission in the third trimester and four other patients were improved, judged by the swollen joint count. (fig lA). One patient had disease onset at the start of her first pregnancy, and an additional patient entered pregnancy with active arthritis in large joints. Both patients continued to have active disease during pregnancy and needed repeated intraarticular injections of corticosteroids in actively inflamed joints (fig 1D). Aggravation of disease activity in six patients with RA was detected at 6 and 12 weeks post partum, decreasing after starting disease modifying antirheumatic drugs (DMARDs) and/or prednisone (figs IA and B). One patient with RA remained in the pregnancy induced remission throughout the observation period. The two patients with RA with active disease during pregnancy improved after the start of DMARDs post partum (fig ID).

\section{Patients with AS}

Figure 2 shows the disease course of the nine patients with AS and their 10 pregnancies by the DAI (A), the BASDAI (B), the patient global score (C), and morning stiffness (D). Except for two patients with minimal disease activity at study entry, a varying disease course with active disease in the first and even more pronounced in the second trimester was seen in eight pregnancies (figs $2 \mathrm{~A}-\mathrm{D}$ ). No remission occurred, but four patients with AS showed a $20 \%$ improvement of disease activity according to ASAS response criteria from the first to the third trimester. Pain scores (item 2 of the BASDAI) (fig 2C) and scores for morning stiffness (fig 2D) were highest in the second and lowest in the third trimester. Morning stiffness disappeared in seven patients with AS in the third trimester. Night pain scores changed in an 

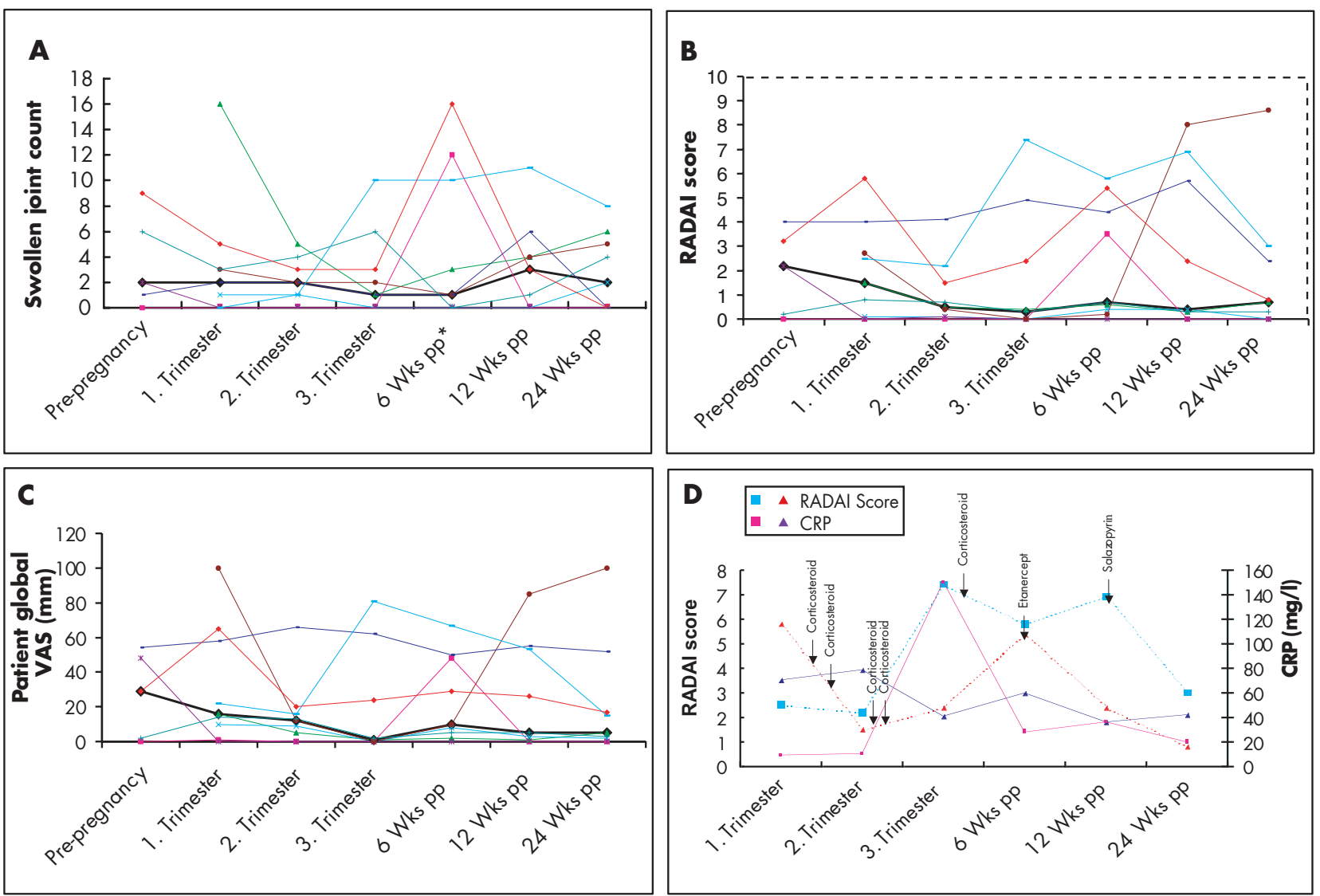

Figure 1 Disease activity in nine patients with RA before, during, and after pregnancy. Each patient is depicted by an individual symbol and colour which is kept in all figures (A-D). The thick, solid line in figs IA, B, and C is the median value for the group. (A) Swollen joint count; (B) RADAl score; (C) patient's global assessment; (D) the RADAl score and CRP measurements of the two patients with RA with active disease during pregnancy. Arrows depict the time of either intra-articular injection of corticosteroids or start of disease modifying treatment. *wks pp, weeks post partum.

unpredictable manner during the observation period (not shown). A linear increase of self assessment scores was seen in all but one patient after delivery (figs 2B-D).

When the median values of the patient and physician global scores were compared a higher disease activity was seen in the patients with AS than in the patients with RA at all times (fig 3A and B). When analysis was carried out specifically for the patient's assessment of pain (item 2 of the BASDAI and item 3 of the RADAI), continuously higher pain scores were found in the patients with AS (fig 3C). This was also reflected by the more extensive use of NSAIDs and paracetamol in the patients with AS than in those with RA (fig 3D).

\section{Disease activity scores}

The swollen joint count correlated well with the RADAI, the coefficient varying between 0.43 and 0.87 . The BASDAI and DAI correlated, on average, with a coefficient of 0.5 . Omitting the question on fatigue, resulted in a 0.5-1.5 reduction of the BASDAI score, mainly during the first trimester, the time when the fatigue of pregnancy was prominent. For the functional indices, a high correlation coefficient $(>0.7)$ between the RADAI and the HAQ was found, whereas correlation between the BASDAI and the DFI was weak $(<0.3)$. None of the patients with RA had any significant functional impairment at study entry, and the changes of HAQ scores at different times were all minimal and not significant $(<0.17)$ (data not shown). The question: Can you sleep in the prone position? of the DFI was given a negative response by all patients with AS in the third trimester. Likewise, bending was difficult for about one third of pregnant patients with RA and AS in late pregnancy, resulting in some impairment reflected in the functional indices. The physician global score was in general lower than the patient global score (fig 3B). The correlation coefficient between patient and physician global scores varied between 0.7 and 0.03 in patients with RA, but was consistently high $(>0.7)$ in patients with AS.

\section{Laboratory}

A slight decrease of thrombocyte numbers and haemoglobin and an increase in leucocyte counts was seen in all patients during pregnancy with a reversal of these changes after pregnancy (data not shown). CRP was normal in all patients with RA with low disease activity during pregnancy, but increased at the postpartum disease flare in three patients. The two patients with very active RA had raised levels of CRP throughout the study (fig 1D). Increases in CRP were seen sporadically in three patients with AS at times of active disease.

\section{Drug treatment}

Acute arthritis in patients with RA was treated with intraarticular corticosteroid injections (fig 1D). For persistent pain and stiffness, low dose prednisone (three patients), NSAID at standard doses (until gestational week 32), and paracetamol were given (fig 3D). Sulfasalazine was continued in four patients with RA in the first trimester, in three during the 

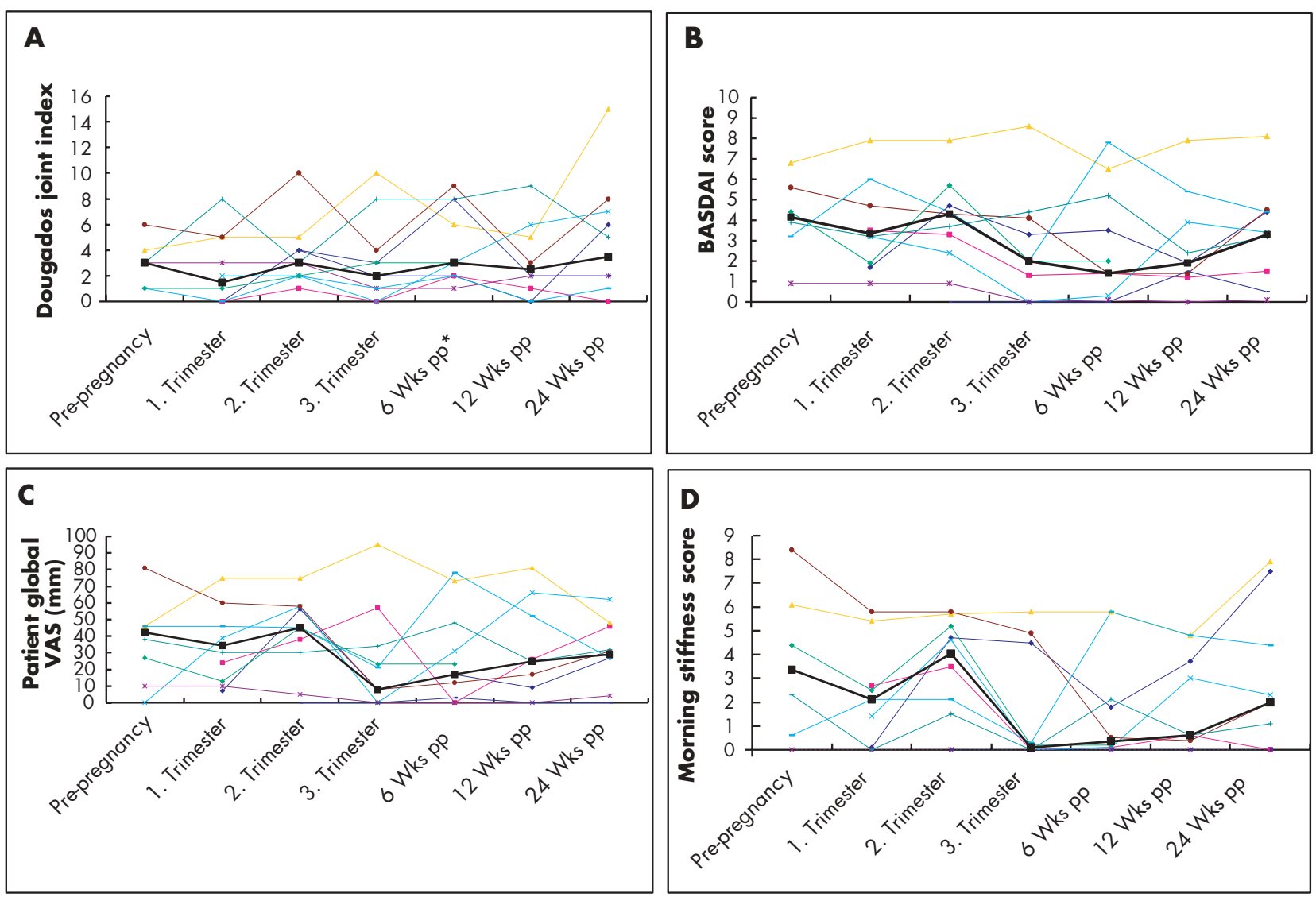

Figure 2 Disease activity in nine patients with AS before, during, and after 10 pregnancies. Each patient is depicted by an individual symbol and colour which is kept in all figures. The thick, solid line in figs 2A-D is the median value for the group. (A) Dougados joint index; (B) BASDAl score; (C) patient's global assessment; (D) morning stiffness score (items 5 and 6 of the BASDAl). "wks pp, weeks post partum.

second, and in two patients with RA also in the third trimester. Signs of postpartum increasing disease activity were immediately treated with appropriate drugs (DMARD in five patients with RA, NSAID in six patients with AS) except for the patient refused owing to breast feeding.

\section{Pregnancy outcome}

Seventeen patients had uneventful pregnancies and delivered healthy infants at term (table 2). Child health remained unremarkable throughout the first 6 months of life. One patient with AS with two consecutive pregnancies had a stillbirth at gestational week 24 in her second pregnancy (reason unknown).

\section{DISCUSSION}

In this study we made a detailed analysis of the course of RA and AS throughout pregnancy and up to 24 weeks after delivery. For the first time, this was done by several validated clinical instruments for assessment of disease activity. We confirmed that AS is active also during pregnancy; however, a mitigation of symptoms occurred in the third trimester. Despite regularly withdrawing NSAIDs at week 32, whenever possible, pain decreased and morning stiffness also disappeared in late gestation in most patients with AS. This was not obvious in our own previous prospective studies of pregnant patients with AS, where we placed the emphasis on physician dependent assessments like metrology. ${ }^{43}$

Search for an explanation of the overall higher disease activity in patients with AS by analysis of singular items recorded in the RADAI and the BASDAI showed that patients with AS had more pain than patients with RA and, consequently, had a higher consumption of pain relieving drugs. Analysis of the pain components in the RADAI showed that the patients with RA had few painful joints and lower pain intensity. In previous studies of non-pregnant patients, a lower pain threshold than in healthy people has been found in patients with RA, but not in patients with AS. ${ }^{25}{ }^{26}$ However, pain induced by pressure as in the two cited studies is not identical to the pain arising from inflammation. The question remains if RA is more profoundly altered or whether the inflammation of AS is not sufficiently suppressed during pregnancy. Most patients with RA entered pregnancy in a state of mild disease, in contrast with the majority of patients with AS who had active disease. Active disease has been shown to be a predictor for persisting activity also in pregnancy. $^{8}$

It is difficult to clarify if the increased pain intensity, particularly in the first and second trimester, experienced by the pregnant patients with AS is inflammatory or due to mechanical alterations. The normal CRP measurements in most patients with AS argue against inflammation. A change of posture and of spinal mobility takes place during pregnancy, ${ }^{27}$ partly as a result of the rise of relaxin with relaxation of the ligaments. ${ }^{28}$ Other hormonal changes that may have a role are the marked increase of progesterone, oestriol, and cortisol during gestation. Owing to progesterone and oestrogen receptors, mechanical properties of pelvic ligaments change in response to sex steroids. ${ }^{29}$ In addition, raised levels of free cortisol may suppress inflammation. The pain relief of the patients with AS in the third trimester is in contrast with the course of non-inflammatory low back pain present in about $50 \%$ of healthy pregnant women, who are 

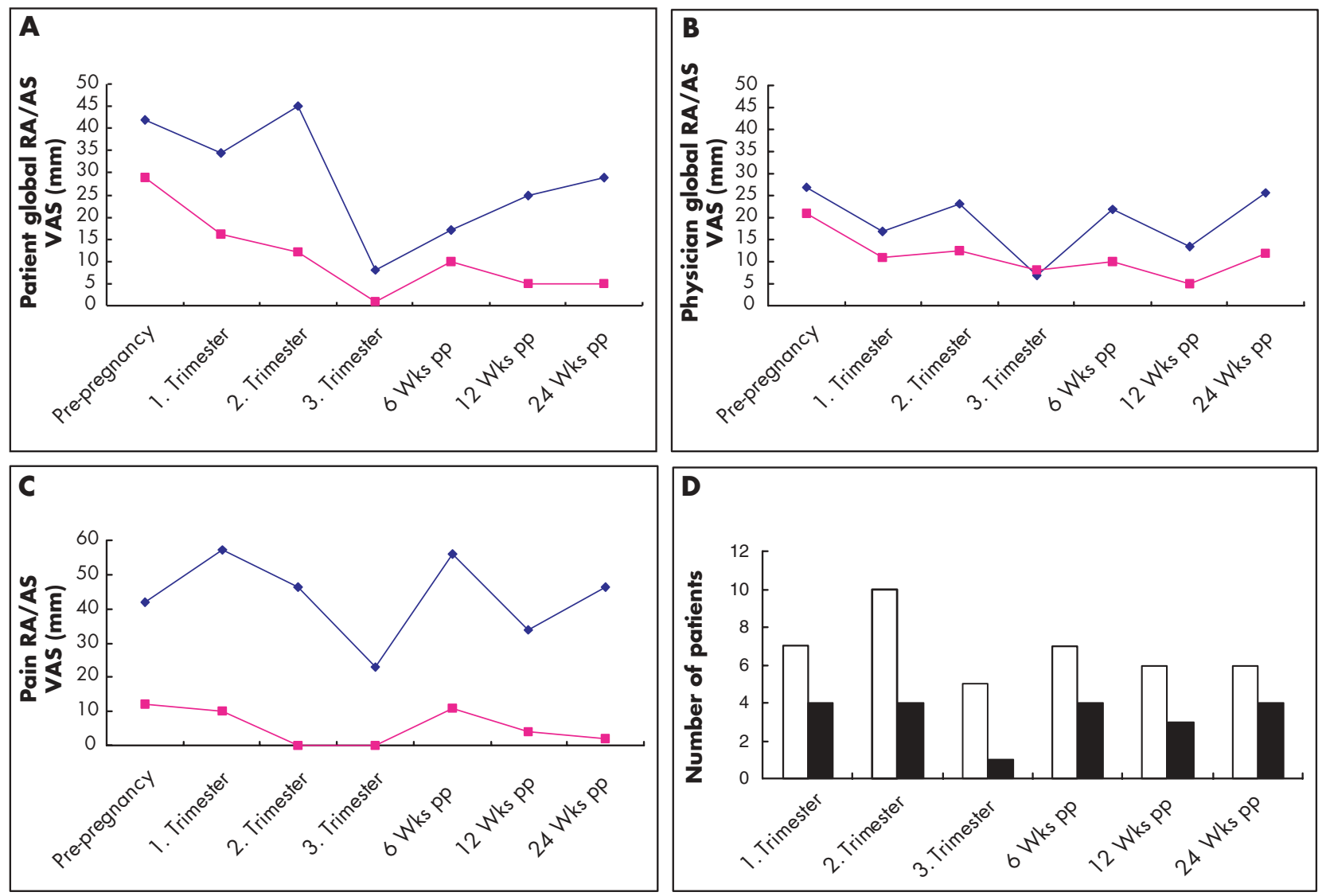

Figure 3 Comparison of disease activity indices in nine patients with RA (red line) and nine patients with AS (blue line) during and after pregnancy. Median values of the patient's global score (A), the physician global score (B), and a pain score (C) are given. The pain score for patients with RA is item 3 of the RADAI: How strong is your arthritis pain today? and item 2 of the BASDAl for patients with AS: How would you describe the overall level of AS neck, back, or hip pain you have had? (D) Consumption of NSAID or paracetamol, or both, in patients with RA (filled blocks) and AS (open blocks) during the observation period.

worse in the later stages of pregnancy. ${ }^{30}$ Furthermore, the surrogate marker of inflammatory activity, morning stiffness, was at its lowest level in the third trimester.

Pregnancy induces profound changes in the maternal body manifested at different stages of pregnancy. The assessment of disease activity is therefore not straight forward, but has to consider symptoms related to the physiology of pregnancy like raised ESR, fatigue, and the increasing burden of the gravid uterus. None of the validated scores for evaluation of disease activity in RA or AS has been adapted for use in pregnancy. In RA, the swollen joint count is an objective measurement of the extent of arthritis and is not confounded by the musculoskeletal pain which may arise spontaneously in pregnancy. ${ }^{31}$ Objective assessment of pregnant patients

Table 2 Pregnancy outcome and breast feeding in nine patients with RA and nine patients (10 pregnancies) with AS

\begin{tabular}{lll}
\hline Characteristics & RA & AS \\
\hline Nulliparous/1 prior birth & $6 / 3$ & $6 / 4$ \\
Pregnancy loss & 1 Miscarriage & 1 Stillbirth \\
Delivery at gestational week & $39(37-41)$ & $39(38-42)^{*}$ \\
Vaginal delivery & 6 & 9 \\
Caesarean section & 3 & 1 \\
Boys/girls & $4 / 5$ & $9 / 1$ \\
Birth weight (g) & $3120(2540-4455)$ & $3130(2870-3755)$ \\
Breast feeding & 7 & 9 \\
\hline \multirow{2}{*}{ *Except for one patient with stillbirth at gestational week 24. }
\end{tabular}

with AS is more difficult because pregnancy itself can induce low back pain or pelvic pain. ${ }^{30}$ In addition, clinical assessments of AS largely depend on the spine, rendering them inaccurate when the effects of the gravid uterus are ignored.

The late stages of pregnancy are accompanied by reduced physical functioning also in healthy women. ${ }^{32}$ Our study indicates that functional indices are not reliable instruments in late pregnancy and need adjustment when used for monitoring pregnant patients. RADAI and BASDAI are disease-specific and include the main symptoms of RA and AS. The results of our study indicate that they are useful instruments for monitoring patients throughout pregnancy and post partum. From our point of view, omission of fatigue from the BASDAI does not increase the reliability of this instrument in pregnant patients. However, patient related instruments may be subject to misinterpretation as seen in one of our patients with RA. Therefore, combination with an objective assessment instrument should be made whenever possible. Addition of composite assessments like the patient global and the physician global score has the advantage of being applicable for both RA and AS, though it may not increase the information already included in the RADAI and BASDAI, respectively. Interestingly, the patient's evaluation differed from the physician's evaluation, reflecting the difference between suffering and observing a disease.

Our study is limited by the small number of patients included and the individual variation of disease activity in each cohort. As a result, by setting a significance level of 5\% and applying the Bonferroni correction, no statistically 
significant change of the disease course over the different times measured was found. However, this was balanced by the detailed clinical assessment of each patient, and the fact that more than half of the patients also had a pre-pregnancy assessment.

In conclusion, higher disease activity as measured by patient and physician dependent instruments was found in pregnant patients with AS than in patients with RA. This difference was partly due to the more pronounced experience of pain in the patients with AS. The disease course in patients with RA most often showed a continuous trend over time as pregnancy progressed, with sustained or increased improvement. By contrast, AS measures did not show this continuity, instead often demonstrating a second trimester increase of disease activity. The results of our study indicate that RA can be monitored during and after pregnancy by the swollen joint count and the RADAI without major interference from pregnancy related symptoms. By contrast, more work needs to be done to create assessment instruments suitable for monitoring pregnant patients with AS.

\section{ACKNOWLEDGEMENTS}

We thank Tiziana Russo and Manuel Bertschy from the Department of Mathematical Statistics and Actuarial Science, University of Berne, for the statistical analysis.

The study was supported by grant 31-59979.00 from the Swiss National Research Fund and by a grant from the Swiss League against Rheumatism.

\section{Authors' affiliations}

M Østensen, L Fuhrer, R Mathieu, M Seitz, P M Villiger, Department of Rheumatology and Clinical Immunology/Allergy, University Hospital of Berne, Berne, Switzerland

\section{REFERENCES}

1 Hench PS. The ameliorating effect of pregnancy on chronic atrophic (infectious rheumatoid) arthritis, fibrositis, and intermittent hydrarthrosis. Proc Staff Meet Mayo Clinic 1938;13:161-7.

2 Ostensen M, Husby G. Ankylosing spondylitis and pregnancy. Rheum Dis Clin North Am 1989;15:241-54.

3 Neely NT, Persellin RH. Activity of rheumatoid arthritis during pregnancy Texas Med 1977;73:59-63.

4 Østensen M, Husby G. A prospective clinical study of the effect of pregnancy on rheumatoid arthritis and ankylosing spondylitis. Arthritis Rheum 1983;26:1155-9.

5 Unger A, Kay A, Griffin AJ, Panayi G. Disease activity and pregnancy associated alpha2-glycoprotein in rheumatoid arthritis during pregnancy. BMJ 1983;286:750-2

6 Nelson JL, Hughes KA, Smith AG, Nisperos BB, Branchaud AM, Hansen JA. Maternal-fetal disparity in HLA class II alloantigens and the pregnancyinduced amelioration of rheumatoid arthritis. N Engl J Med 1993:329:466-71.

7 Quinn C, Mulpeter K, Casey EB, Feighery CF. Changes in levels of IgM RF and $\alpha 2$ PAG correlate with increased disease activity in rheumatoid arthritis during the puerperium. Scand J Rheumatol 1993;22:273-9.

8 Ostensen M, Ostensen $\mathrm{H}$. Ankylosing spondylitis - the female aspect. J Rheumatol 1998;25:120-4.

9 Gran JT, Ostensen M. Spondylarthritides in females. Ballieres Clin Rheumatol $1998 ; 12: 695-715$.
10 Camp AV. An articular index for the assessment of rheumatoid arthritis. Orthopaedics (Oxford), 1971;4:39-45.

11 Hawley DJ, Wolfe F. Sensitivity to change of the Health Assessment Questionnaire (HAQ) and other clinical and healthy status measures in rheumatoid arthritis: results of short-term clinical trials and observational studies versus long-term observational studies. Arthritis Care Res 1992;5:130-6.

12 Meenan RF. Assessing therapeutic effectiveness in the rheumatic diseases: the case for functional health status measures. In: Paulus $\mathrm{HE}$, Erlich $\mathrm{AE}$, Lindenlaub $\mathrm{E}$, eds. Controversies in the evaluation of analgesic antiinflammatory anti-rheumatic drugs. New York: Schatauer Verlag, 1981:353-64.

13 Ostensen M. Pregnancy in psoriatic arthritis. Scand J Rheumatol 1988;17:67-70.

14 Barreft JH, Brennan P, Fiddler M, Silman AJ. Does rheumatoid arthritis remit during pregnancy and relapse postpartum? Results from a nationwide study in the United Kingdom performed prospectively from late pregnancy. Arthritis Rheum 1999;42:1219-27.

15 Alavi A, Arden N, Spector TD, Axford JS. Immunoglobulin G glycosylation and clinical outcome in rheumatoid arthritis during pregnancy. $J$ Rheumatol 2000;27:1379-85.

16 Arnett FC, Edworthy SM, Bloch DA, McShane DJ, Fries JF, Cooper NS, et al. The American Rheumatism Association 1987 revised criteria for the classification of rheumatoid arthritis. Arthritis Rheum 1988;31:315-24.

17 Dougados M, van der Linden S, Juhlin R, Huiffeldt B, Amor B, Calin A, et al. The European Spondylarthropathy Study Group: preliminary criteria for the classification of spondylarthropathy. Arthritis Rheum 1991;34:1218-27.

18 Stucki G, Liang MH, Stucki S, Bruhlmann P, Michel BA. A self-administered rheumatoid arthritis disease activity index (RADAl) for epidemiological research. Arthritis Rheum 1995;38:795-8.

19 Prevoo ML, Kuper IH, van't Hof MA, van Leeuwen MA, van de Putte LB, van Riel PL. Modified disease activity scores that include twenty-eight joint counts. Development and validation in a prospective longitudinal study of patients with rheumatoid arthritis. Arthritis Rheum 1995;38:44-8.

20 Garrett S, Jenkinson T, Kennedy LG, Whitelock H, Gaisford P, Calin A. A new approach to defining disease status in ankylosing spondylitis: the Bath Ankylosing Spondylitis Disease Activity Index. J Rheumatol 1994;21:2286-91.

21 Dougados M, Gueguen A, Nakache JP, Nguyen M, Armor B. Evaluation of a functional index and an articular index in ankylosing spondylitis. J Rheumatol 1988;15:302-7.

22 Hultgren S, Broman JE, Gudbjörnsson B, Hetta J, Lindqvist U. Sleep disturbances in outpatients with ankylosing spondylitis - a questionnaire study with gender implications. Scand J Rheumatol 2000;29:365-9.

23 Pinals RS, Masi AT, Larsen RA. Preliminary criteria for clinical remission in rheumatoid arthritis. Arthritis Rheum 1981;24:1308-15.

24 Anderson JJ, Baron G, van der Heijde D, Felson DT, Dougados M. Ankylosing spondylitis assessment group preliminary definition of short-term improvement in ankylosing spondylitis. Arthritis Rheum 2001;44:1876-86.

25 Gerecz-Simon EM, Tunks ER, Heale JA, Kean WF, Buchanan WW. Measurement of pain threshold in patients with rheumatoid arthritis, osteoarthritis, ankylosing spondylitis, and healthy controls. Clin Rheumatol 1989;8:467-74.

26 Incel NA, Erdem HR, Ozgocmen S, Catal SA, Yorgancioglu ZR. Pain pressure threshold values in ankylosing spondylitis. Rheumatol Int 2002;22:148-50.

27 Gilleard W, Crosbie J, Smith R. Effect of pregnancy on trunk range of motion when sitting and standing. Acta Obstet Gynecol Scand 2002;81:1011-20.

28 Goldsmith LT, Weiss G, Steinetz BG. Relaxin and its role in pregnancy. Endocrinol Metabol Clin North Am 1995;24:171-86.

29 Reay Jones NH, Healy JC, King U, Saini S, Shousha S, Allen-Mersh TG. Pelvic connective tissue resilience decreases with vaginal delivery, menopause and uterine prolapse. Br J Surg 2003;90:466-72.

30 Hainline B. Low-Back pain in pregnancy. In: Devinsky O, Feldmann E, Hainline B, eds. Neurological complications of pregnancy. New York: Raven Press, 1994:65-76

31 Ireland ML, OH SM. The effects of pregnancy on the musculoskeletal system. Clin Orthop 2000;372:169-79.

32 McKee MD, Cunningham M, Jankowski KRB, Zayas L. Health-related functional status in pregnancy: relationship to depression and social support in a multi-ethnic population. Obstet Gynecol 2001;97:988-93. 